Research Article

\title{
Damage Detection Applied to a Full-Scale Steel Bridge Using Temporal Moments
}

\author{
Bjørn T. Svendsen $(\mathbb{D}$, Gunnstein T. Frøseth $(\mathbb{D}$, and Anders Rönnquist $(\mathbb{D}$ \\ Department of Structural Engineering, Norwegian University of Science and Technology, Trondheim 7491, Norway \\ Correspondence should be addressed to Bjørn T. Svendsen; bjorn.t.svendsen@ntnu.no
}

Received 6 November 2019; Accepted 18 January 2020; Published 27 February 2020

Academic Editor: Mahmoud Bayat

Copyright (C) 2020 Bjørn T. Svendsen et al. This is an open access article distributed under the Creative Commons Attribution License, which permits unrestricted use, distribution, and reproduction in any medium, provided the original work is properly cited.

\begin{abstract}
The most common damages in existing highway and railway steel bridges are related to fatigue and are, as reported in the literature, found in the structural system of the bridge deck. This paper proposes a methodology for detecting damaged joint connections in existing steel bridges to improve the quality of bridge inspections. The methodology combines the use of temporal moments from response measurements with an appropriate instrumentation setup. Damaged joint connections are identified by comparing statistical parameters based on temporal moments to a baseline, where the baseline data are established from statistical parameters evaluated for all considered joint connections. Localization of damaged joint connections is performed by utilizing the instrumentation setup. The feasibility of the proposed methodology is demonstrated through an experimental study on a full-scale steel riveted truss bridge with two known damages below the bridge deck, where both damages are identified and localized. The proposed methodology can improve the identification of critical structural damage during bridge inspections and is applicable to open-deck steel bridges.
\end{abstract}

\section{Introduction}

Deterioration and ageing of infrastructure is a major concern worldwide. Many highway and railway bridges are subject to increasing demands with respect to traffic loads and intensity, even though these structures are approaching or have exceeded their original design life. Considering the requirements for more efficient transportation systems, for which these bridges were not originally designed, many bridges are still in service despite ageing and the associated damage accumulation.

Many of the existing bridges built in the first half of the 20th century in Europe and the U.S. are made of steel. The primary damage mechanism in these bridges is fatigue, and the most common types of fatigue damage reported are found in the structural system of the bridge deck [1]. Consequently, several case studies are performed on service life estimation and fatigue reliability analysis of structural components in the bridge deck structural system of steel bridges [2-9]. The connections between longitudinal stringers and transverse girders are critical and have been subject to investigation in studies of railway bridges [10-16]. These stringer-to-girder connections are not easily accessible and are consequently difficult to inspect. The induced damages involve cracking in various parts of the connections and can, if not detected at an early stage, develop and lead to component failure being critical for the structural integrity.

Inspections are performed to ensure the short-term safe operation of bridges. For railway bridges, visual inspection is the preferred nondestructive testing (NDT) method [17]. Typically, these inspections are specified in regular intervals to establish maintenance needs. There are several challenges related to visual inspections. First, inspections require direct access to critical structural components. Many bridges are in remote locations and have low general accessibility. To perform a full inspection requires either operational downtime or temporary installation of access support or both. Second, the quality of the inspection depends on the experience and knowledge of the inspector. Fatigue damage 
can be difficult to detect due to low visibility. Critical structural damage can be difficult to establish until the structure is subjected to operational or strong environmental loading. As such, having full access to a bridge for inspection is costly and provides no guarantee of finding damage. Third, periodic inspections do not provide full up-to-date information about the current state of the bridge condition. Structural health monitoring (SHM), defined as the process of implementing an automated and online strategy for damage detection in a structure [18], can provide such information. Although SHM systems can undoubtedly optimize the inspection process, development is still needed to ensure that such systems are affordable and reliable and that these systems are likely to detect both local and global damages. With the large number of existing bridges in infrastructure, an enhanced inspection methodology is needed to detect damage during bridge inspections.

Many vibration-based damage detection methods exist, of which most applications are based on numerical studies or laboratory studies performed in a controlled environment [19-23]. One method not widely reported in the literature is the use of temporal moments [24]. Temporal moments can be used to characterize shock or transient dynamic signals and are useful in describing the shape of such time histories. Only one study is found that has applied this method for damage detection purposes. Hemez and Doebling [25] applied temporal moments to acceleration response measurements obtained from a complex threaded assembly of metallic and joint components, where one of the objectives was to distinguish a loose assembly test from several tight assembly tests. The load was established as patches of explosives on the external surface of the system. The study concluded that the loose assembly test could be successfully distinguished from other tests through analyses using temporal moments. A similar analysis approach is utilized to detect damage in joint connections of full-scale steel bridges.

In this paper, a new methodology is proposed to detect damage in stringer-to-girder connections from the bridge deck to improve the process and quality of bridge inspections. This methodology consists of combining the use of temporal moments from response measurements with an appropriate instrumentation setup and a systematic monitoring procedure. As such, an experimental study on a fullscale steel bridge is carried out. Instrumentation of the bridge deck is performed, and acceleration response measurements are obtained using a modal hammer. By applying the method of temporal moments to the transient part of the acceleration response, feature vectors containing statistical parameters are established for the stringer-to-girder connections. Damaged connections are identified and localized by (1) investigation of individual statistical parameters and (2) establishing a damage indicator matrix by comparing feature vectors using a correlation analysis. The effect of sampling frequency is also investigated. The feasibility of the proposed methodology is discussed with respect to its applicability to similar bridges in service, in particular as a part of a general inspection plan for damage detection.

\section{Temporal Moments}

2.1. Temporal Moments in Continuous Time Signals. Temporal moments describe how the energy of a signal is distributed over time. These are established by considering the square of the signal amplitudes and provide an alternative to statistically characterizing transient signals [26]. The $i$ th-order temporal moment, $M_{i}$, about a reference time, $t_{r}$, for a continuous system is defined as [24]

$$
M_{i}\left(t_{r}\right)=\int_{-\infty}^{+\infty}\left(t-t_{r}\right)^{i} y^{2}(t) \mathrm{d} t
$$

where $t$ denotes the time and $y(t)$ is the system output, which is typically the response measurement signal. The first five moments are of particular relevance to describe the statistical properties of a transient signal: energy $(E)$, central time $(T)$, mean-square duration $\left(D^{2}\right)$, skewness $\left(S_{t}^{3}\right)$, and kurtosis $\left(K_{t}^{4}\right)$. These moments are expressed in terms of basic and central moments. A basic moment is defined when the reference time, $t_{r}$, is 0 . A simplified notation can then be introduced as

$$
M_{i}(0)=M_{i}
$$

Furthermore, a central moment, $M_{i}(T)$, is defined about a value $T$ of the reference time when the first-order temporal moment is zero, i.e.,

$$
M_{1}(T)=0 .
$$

The definitions of the first five moments about $T$ are given in terms of the basic and central moments. The energy, $E$, is the zero-order moment defined as

$$
E=M_{0}(T)=M_{0}
$$

where $M_{0}(T)$ is the zero-order temporal moment. This moment is defined as the integral of the signal squared. It is independent of any reference time. Thus, it is referred to as the energy of the signal. The central time, $T$, is the first normalized central moment, which is defined as

$$
T=\frac{M_{1}}{E},
$$

where the basic moment, $M_{1}$, is established from the first-order temporal moment, $M_{1}(T)$, using equations (1) and (2):

$$
M_{1}(T)=\int_{-\infty}^{+\infty}(t-T) y^{2}(t) \mathrm{d} t=0
$$

The normalization with respect to $E$ provides the time where the centroid of the energy is located, i.e., the centroid of the area under the squared signal amplitudes. By considering the distribution of energy over time, this moment represents the point where half of the energy has passed and half is to arrive at the sensor. A similar normalization is provided for the higher moments. The mean-square duration, $D^{2}$, is the second normalized central moment defined as

$$
D^{2}=\frac{M_{2}(T)}{E}
$$


where $M_{2}(T)$ is the second-order temporal moment defined according to equation (1). The mean-square duration describes the dispersion of the energy in the signal about the central time, $T$. The root-mean-square duration, $D$, is obtained by taking the square root of the expression in equation (7). Due to the normalization, this expression provides the time of the energy dispersion. The most significant part of the energy is expected to be around the central time. The mean-square and root-mean-square (RMS) durations are analogous to the variance and standard deviation of regular statistical moments, respectively. The central skewness, $S_{t}$, is the third normalized central moment defined as

$$
S_{t}^{3}=\frac{M_{3}(T)}{E},
$$

where $M_{3}(T)$ is the third-order temporal moment defined according to equation (1). Skewness normalized by the RMS duration provides a nondimensional measure and is defined as

$$
S=\frac{S_{t}}{D}
$$

Skewness describes the shape of the signal energy in terms of symmetry. Symmetry about the central time T, or centroid, indicates zero skewness. A transient signal with high amplitudes to the left and a corresponding low-amplitude tail on the right side of the centroid has a positive skewness. Similarly, the opposite provides a negative skewness. The central kurtosis, $K_{t}$, is the fourth normalized central moment defined as

$$
K_{t}^{4}=\frac{M_{4}(T)}{E},
$$

where $M_{4}(T)$ is the fourth-order temporal moment defined according to equation (1). Like skewness, kurtosis normalized by the RMS duration provides a nondimensional measure and is defined as

$$
K=\frac{K_{t}}{D} .
$$

Kurtosis describes the tail shape of the signal energy. More precisely, kurtosis provides a measure for the outliers in the signal that are represented in the tails of the area under the squared signal amplitudes. In general, a low value of kurtosis indicates few and less extreme outliers, whereas a high value indicates several and more extreme outliers. Hence, a high value of kurtosis indicates more area in the tails. The root energy amplitude, $A_{E}$, is an alternative way to describe the energy and is defined as

$$
A_{E}=\sqrt{\frac{E}{D}}
$$

This expression is simply the square root of the energy normalized by the RMS duration. Table 1 summarizes the central moments in terms of the temporal moments.

The following two clarifications should be noted. First, a central moment is simply a temporal moment with respect to the reference time $T$ when the first-order temporal moment is zero. All central moments of order 1 through 4 are normalized by the basic moment $E$, which is the zeroorder temporal moment. Consequently, the units become seconds. Additionally, a second normalization by the RMS duration, $D$, provides an alternative measure of the thirdand fourth-order central moments with nondimensional units. Second, considering the definition provided in equation (1), it is obvious that the integral of the signal squared is the total energy of the signal, or the area of the defined signal squared. The signal squared represents a distribution, which is analogous to a probability density function in regular statistical theory. Hence, the central moments are statistical moments of this distribution, also representing how the energy of the signal is distributed in time.

2.2. Temporal Moments in Discrete Time Signals. The ithorder temporal moment defined by equation (1) about the central time $T$ can be approximated for a discrete signal of finite duration as

$$
M_{i}(T) \approx \frac{1}{2} \sum_{j=0}^{N-2}[\Delta t(j+0.5)-T]^{i} \Delta t\left[y_{j}^{2}+y_{j+1}^{2}\right],
$$

where $\Delta t$ is the time increment between two response measurements and the sample index is defined to run from 0 to $N-1$ as commonly used in digital signal processing. Furthermore, the general integral

$$
\int_{-\infty}^{+\infty} y^{2}(t) \mathrm{d} t \approx \sum_{j=0}^{N-2} \frac{\Delta t}{2}\left(y_{j}^{2}+y_{j+1}^{2}\right),
$$

is based on the trapezoidal rule. The application of equation (13) numerically provides the basis for establishing the central and normalized central moments. The normalized central moments in terms of the basic moments provide an alternative way of establishing the five temporal moments and can be utilized with equation (13):

$$
\begin{aligned}
E & =M_{0}, \\
T & =\frac{M_{1}}{M_{0}} \\
D^{2} & =\left(\frac{M_{2}}{M_{0}}\right)-\left(\frac{M_{1}}{M_{0}}\right)^{2}, \\
S_{t}^{3} & =\left(\frac{M_{3}}{M_{0}}\right)-3\left(\frac{M_{2} M_{1}}{M_{0}^{2}}\right)+2\left(\frac{M_{1}}{M_{0}}\right)^{3}, \\
K_{t}^{4} & =\left(\frac{M_{4}}{M_{0}}\right)-4\left(\frac{M_{3} M_{1}}{M_{0}^{2}}\right)+6\left(\frac{M_{2} M_{1}^{2}}{M_{0}^{3}}\right)-3\left(\frac{M_{1}}{M_{0}}\right)^{4} .
\end{aligned}
$$

The derivations of these relationships can be established by considering the temporal moments according to equation (1) about the reference time $T$ in combination with equation 
TABLE 1: Definition of central and normalized central moments.

\begin{tabular}{|c|c|c|c|c|}
\hline Parameter & Moment & Moment order & Definition & Units \\
\hline$E$ & Energy & 0 & $E=M_{0}$ & $\left(\mathrm{~m} / \mathrm{s}^{2}\right)^{2} \mathrm{~s}$ \\
\hline$T$ & Central time & 1 & $T=M_{1} / E$ & $\mathrm{~s}$ \\
\hline$D$ & RMS duration & 2 & $D=\sqrt{M_{2}(T) / E}$ & $\mathrm{~s}$ \\
\hline$S_{t}$ & Central skewness & 3 & $S_{t}=\sqrt[3]{M_{3}(T) / E}$ & s \\
\hline$S$ & Normalized skewness & 3 & $S=S_{t} / D$ & - \\
\hline$K_{t}$ & Central kurtosis & 4 & $K_{t}=\sqrt[4]{M_{4}(T) / E}$ & s \\
\hline$K$ & Normalized kurtosis & 4 & $K=K_{t} / D$ & - \\
\hline$A_{E}$ & Root energy amplitude & - & $A_{E}=\sqrt{E / D}$ & $\left(\mathrm{~m} / \mathrm{s}^{2}\right)$ \\
\hline
\end{tabular}

(2) and the provided definitions of the central moments in Table 1.

For the application of temporal moments in this study, the signals analysed are acceleration response measurements or time histories. However, the application is also valid for the transient part of the response obtained using other measures, such as strain, force, displacement, or velocity [24]. The implementation of temporal moments numerically is made available [27].

2.3. Feature Vectors. The central and normalized central moments given in Table 1 can be established as statistical parameters of a transient signal. In the following, these parameters are also referred to as features, which are defined as quantities established from response measurements that can be used to indicate damage [26]. All features constitute a feature vector, which is defined as

$$
\mathbf{d}_{m}=\left[\begin{array}{llllllll}
E & T & D & A_{E} & S_{t} & S & K_{t} & K
\end{array}\right],
$$

where the subscript $m$ denotes the joint number.

\section{Bridge Description and Experimental Study}

3.1. Bridge Description. The Hell Bridge Test Arena is a fullscale, steel-riveted railway truss bridge taken out of service and moved to foundations on land, as shown in Figure 1. The bridge is $4.5 \mathrm{~m}$ wide and has a total span of $35 \mathrm{~m}$. It serves as a full-scale laboratory for research within SHM, damage detection, bridge inspection and service life estimation. Figure 2 shows a simplified 3D model of the main structural steel in the Hell Bridge Test Arena.

The bridge deck consists of longitudinal stringers connected to transverse girders (floor beams). The stringer-togirder connections are made using double angle connections that are mechanically fastened with rivets. These are designed as shear connections, transferring the stringer end forces to the girder. Two mechanisms are commonly reported in this type of connection [1]: rotation of the stringer ends associated with bending and overlooked interactions between the bridge deck structural system and the main load carrying structure. These mechanisms are generated by deformation-induced secondary effects causing fatigue cracking [11]. In the following sections, the stringer-togirder connections are referred to as both joints and joint connections.

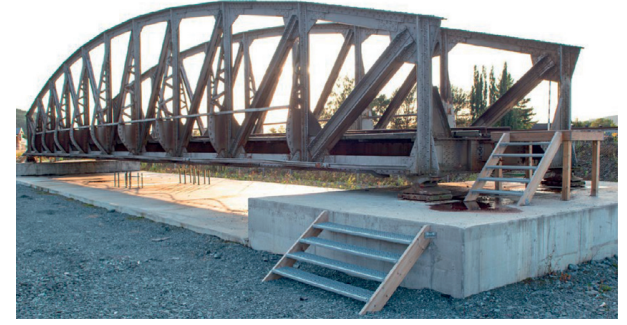

Figure 1: Hell Bridge Test Arena.

There are two known damages in the joint connections in the bridge deck structural system of the Hell Bridge Test Arena. These damages are located on one side of the middle of the bridge, which was retrofitted during the initial part of the bridge lifetime. Despite the retrofit, all joint connections have the same loadbearing function. The damages were not found during routine interval inspections while the bridge was operational. These damages were originally discovered during a measurement campaign prior to the bridge being taken out of service, in which strain measurements were performed only on a selection of the joint connections. The damages result in unwanted vertical movement of the respective stringers when the bridge is subjected to operational loads. Consequently, the result is a severe reduction in the loadbearing capacity.

3.2. Damage Detection Strategy. A structure that can function satisfactorily but is no longer operating in an ideal condition is defined as a damaged structure [28]. In the most basic term, damage is defined as a change in the structural system that affects the performance of the structure [18]. Such a change can be in the material properties, geometry, boundary conditions, or connections of the structural system. Determining the damage state can be accomplished according to a hierarchical structure, or levels, where increased knowledge of the damage state is represented in the given order of levels. These are defined as (1) existence, (2) localization, (3) type, (4) extent, and (5) prediction of damage [28, 29].

Assessing damage requires a comparison between two different states of a system [30]. In this study, all relevant joint connections are investigated to establish damage in the bridge deck. A baseline, representing the normal and undamaged condition, is established from statistical 


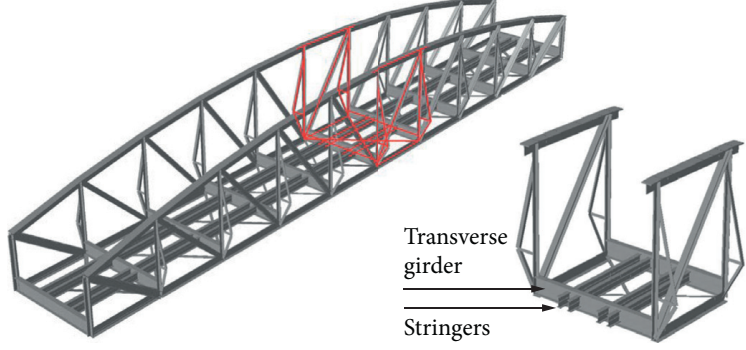

(a)

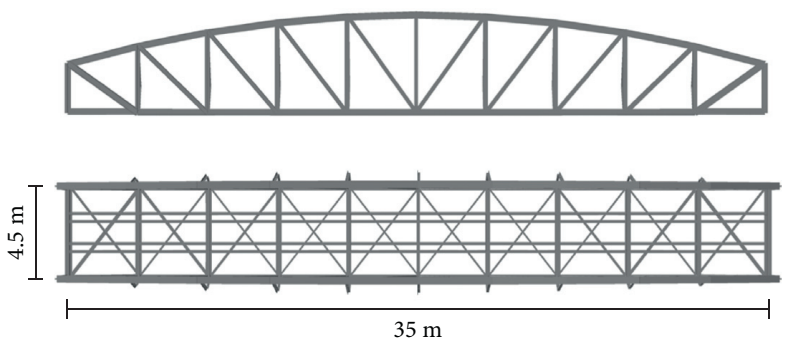

(b)

Figure 2: Simplified 3D model of the main structural steel in the Hell Bridge Test Arena. (a) 3D perspective view including a detailed bridge section. (b) Vertical wall (top) and plan view of the bridge deck (bottom).

parameters using all joints. Damage is then assessed based on the comparison of the results of individual joints to the baseline. As such, damaged joint connections are identified by comparing results to nominally identical connections and localized utilizing the instrumentation setup.

3.3. Experimental Study. An instrumentation setup and systematic monitoring procedure was established in the experimental study. Sensors were placed symmetrically about an impact location to effectively assess the joint connections. Figure 3 shows an overview of the bridge joints subject to investigation and the full bridge deck instrumentation, including all sensor and impact locations.

A symmetric instrumentation setup about the geometric centre of the bridge deck was made using 32 sensor locations, denoted S01-S32, and 9 impact locations, denoted X1-X9. Both sensor and impact locations were accessible from the bridge deck. Altogether, 64 joints, labelled J01-J64, were assessed utilizing the instrumentation setup. This included 8 joints per impact location from $\mathrm{X} 2-\mathrm{X} 8$ and 4 joints per impact location X1 and X9. The known damaged joints in the bridge deck were labelled J29 and J31. The geometric centre of the bridge coincides with the impact location $\mathrm{X} 5$.

The testing was performed using 16 Dytran 3583BT triaxial accelerometers and a PCB large-sledge modal impulse hammer (model 086D50). The accelerometers were mounted at the midspan of the stringers. Data acquisition was performed using a CompactRIO 9036 from National Instruments with 8 input modules (model 9234 C Series Sound and Vibration) at a sampling frequency of $2048 \mathrm{~Hz}$. A minimum of 5 impacts were systematically induced at the predefined impact locations. To obtain a strong transient signal, the testing was performed using a hard-plastic hammer tip (model 084A32), which gave a high-frequency excitation. Measurements were recorded in the $z$-direction only. The preprocessing of the time series mainly consisted of three steps: first, the sensor sensitivity was included; second, the time series were optimized by synchronization using the peak value of each signal and then trimmed to the desired length; and finally, the linear trends were removed by detrending the signals. From the preprocessing, each recording resulted in a time series of $1.0 \mathrm{~s}$, where $0.1 \mathrm{~s}$ was recorded prior to the impact and $0.9 \mathrm{~s}$ was recorded after the impact.
The number of sensors to be applied is not of significant importance. The methodology allows the use of one sensor only as a minimum. Repeated testing by moving the sensors to the predefined sensor locations provides the required measurements. However, to reduce the number of impacts, a minimum of 8 sensors is recommended. This setup also ensures that the maximum number of joints is analysed using the same input, which increases the accuracy of the results.

By utilizing the symmetry of the instrumentation setup and the systematic monitoring procedure, acceleration response measurements can be established with the fewest number of sensors and impacts. This approach provides information about the transmissibility of the signal in the time domain, where the transmissibility represents the signal from the impact location and through the joint to be assessed.

\section{Results}

4.1. General. Acceleration response measurements, together with the average absolute peak acceleration response, provide a basic description of the response from the joints considered. The results from the experimental study are investigated using two approaches: first, the most relevant statistical parameters, i.e. features, are plotted and compared individually for all joints from the acceleration response measurements; second, a damage indicator matrix is established based on the correlation between the feature vectors for each joint analysed.

4.2. Basic Response Description. A comparison of the time histories of the acceleration response measurements for all joints in the middle part of the bridge is shown in Figure 4. All joints connected to the same girder specified by the impact location are presented in the vertical columns, where the response is shown for the same impact. All response measurements show the main transient response for one impact occurring in the time interval of $0.09-0.16 \mathrm{~s}$.

From these plots, a general trend in the response measurements of the joints is observed: each response is characterized by a distinct peak followed by an exponential decay in the signal amplitudes. In general, the curves in each plot follow each other fairly well considering the shape of the 


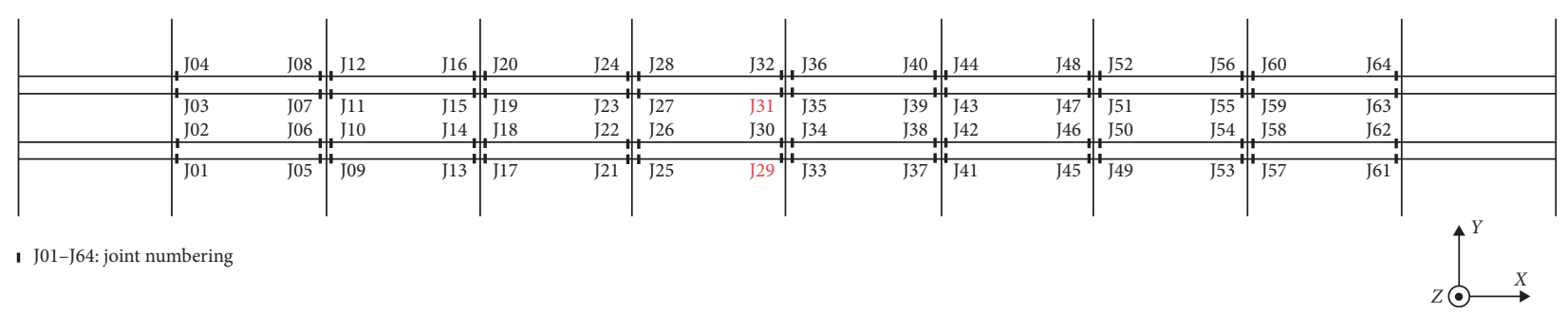

(a)

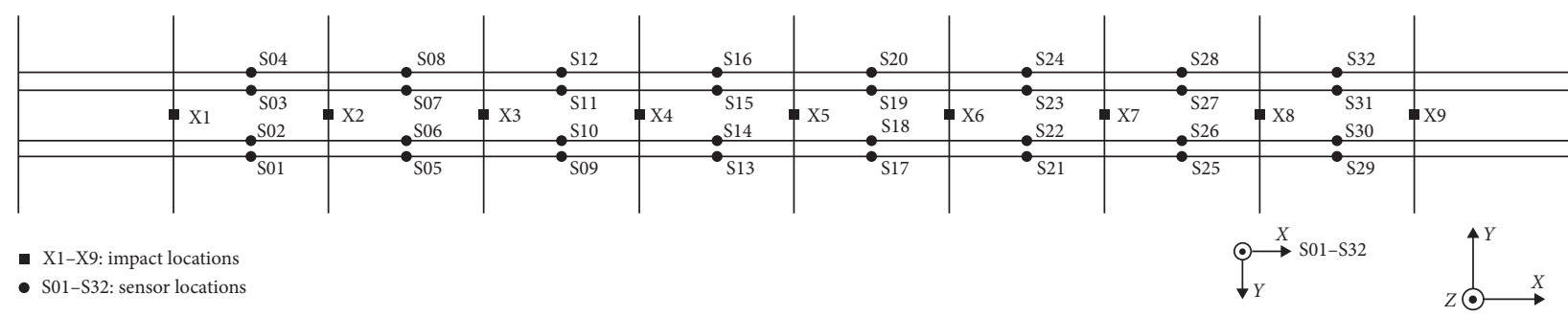

(b)

Figure 3: Instrumentation setup of the Hell Bridge Test Arena bridge deck (plan view). (a) Joint numbering with known damaged joints highlighted in red. (b) Sensor and impact locations.

transient response, including the decay. This finding is expected since most of the joints are in fact assumed to be identical and undamaged. However, exceptions are observed particularly for impact locations X5 and X6. The relevance of these deviations with respect to damage is best evaluated using statistical parameters.

A comparison of all joints for the average absolute peak acceleration response of 5 impacts per impact location is shown in Figure 5. A large variation is observed when comparing all joints and the joints within the same impact location. However, the average peak value for J29 deviates from that of the other joints, indicating that this joint behaves differently than the others.

The average absolute peak value can provide a distinction in the results, but it is not an adequate feature for identifying damage. This inadequacy is mainly due to two reasons. First, this feature is a sensitive measure and is strongly dependent on the sampling frequency, placement of sensors, and input force. For transient vibration, sampling at high frequencies can more reliably capture the actual peak acceleration than sampling at low frequencies. Furthermore, small deviations in sensor placements are common when performing field measurements on large structures. Sensitive features can provide large deviations in results caused by operational and environmental variability and not just from the presence of damage. Second, this feature only provides one measure of a signal that generally contains a vast amount of information.

Making observations that may identify damaged joints is challenging due to the general similarity observed in the acceleration response measurements and the large variability in the average absolute peak acceleration values. Hence, establishing statistical parameters of the signals to quantify characteristics of the acceleration response measurements is needed to provide reliable information and increase confidence in results.
4.3. Statistical Parameters. The results of analysing the main statistical parameters are shown in Figures 6 and 7 by moment order. Energy $(E)$, central time $(T)$, and RMS duration $(D)$ are presented in Figure 6, whereas central skewness $\left(S_{t}\right)$, central kurtosis $\left(K_{t}\right)$, and root energy amplitude $\left(A_{E}\right)$ are presented in Figure 7.

In both figures, each marker in the plots represents the average value obtained from 5 impacts. The short-dashed grey vertical lines separate the impact locations, and all markers within each impact location represent results obtained from the same impacts. The dashed horizontal lines represent the upper and lower outlier limits. To find these limits, the interquartile range (IQR) for each of the data sets with the statistical parameters is established. The IQR is a measure of variability and divides the data considered into quartiles. The IQR is the range between the 75th and 25th percentiles of the data and consequently contains the middle portion or $50 \%$. The outlier limits are defined as 1.5 times the IQR length from the upper and lower percentile, which is considered a common criterion for outliers [31]. Values outside of this range are extreme data values. All plots show both the upper and lower limits, i.e., $U_{\lim }$ and $L_{\text {lim }}$, except for the energy. Here, the lower limit is less than zero, representing a negative area or energy not considered realistic.

For the known damaged joint connections, the most obvious results are obtained for J29. For J29, all statistical parameters except for energy are outliers; however, for energy, J29 has the lowest value of all joints. For J31, outliers are observed for RMS duration, central skewness, and central kurtosis, but otherwise, the statistical parameters are within the IQR.

For the undamaged joint connections, J41 and J45 are represented with outliers in energy and central time but otherwise have values well within the IQR. Joints J33 and J36 have values very close to or exceeding the outlier limits for central skewness and central kurtosis. Additionally, high 

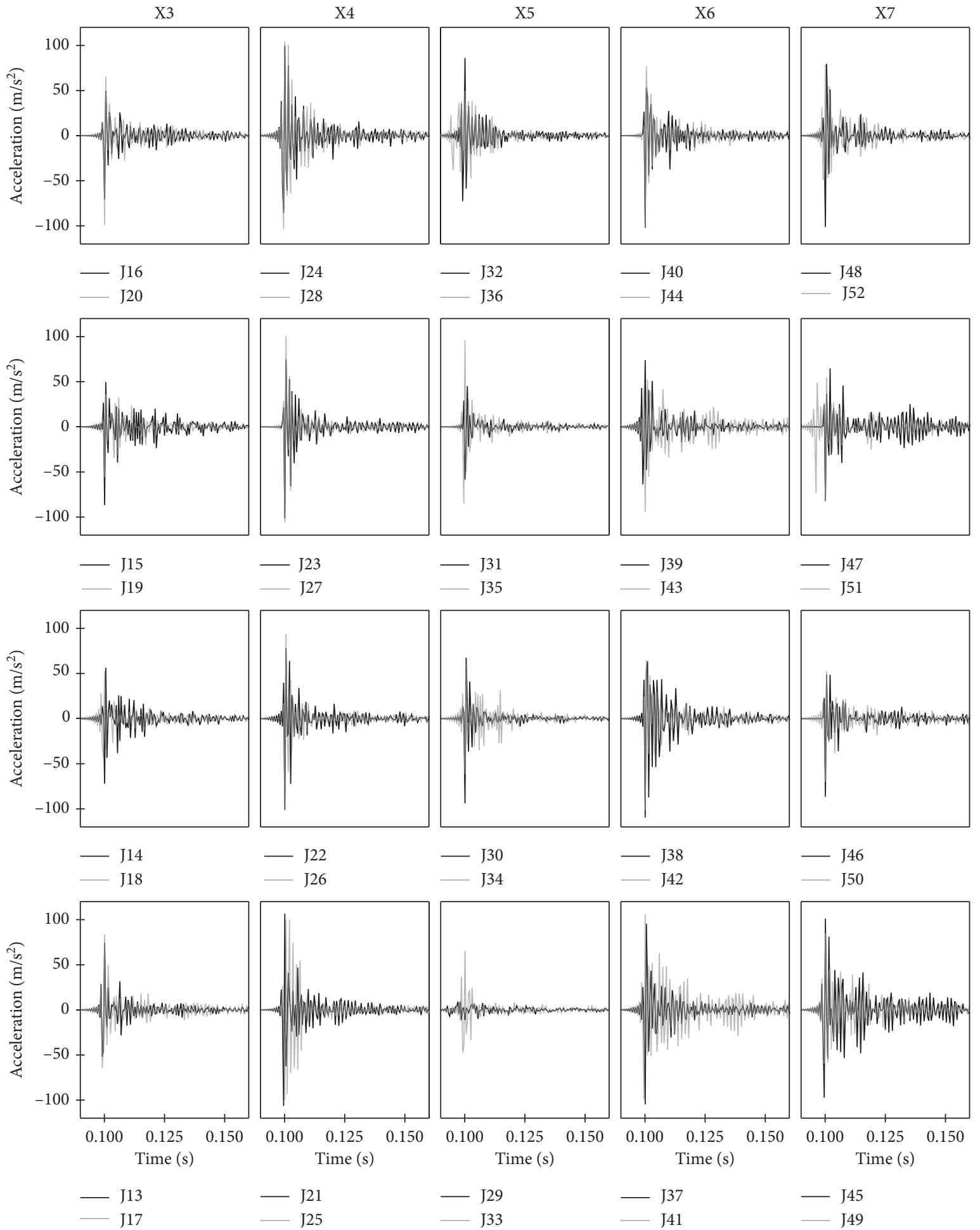

FIgURE 4: Comparison of the acceleration response measurements based on one impact for the joints in the middle part of the bridge.

values are observed for RMS duration. J25 has outliers for energy and root energy amplitude. An interesting observation is made for joints J18 and J19. The statistical parameters are observed to be within the IQR. However, the values are close to the outlier limits for all statistical parameters.
Detailed results of all statistical parameters for the abovementioned joints are summarized in Table 2. Values exceeding the outlier limits are highlighted. Additionally, the lower and upper limits, arithmetic mean, and median values are included in this table. 


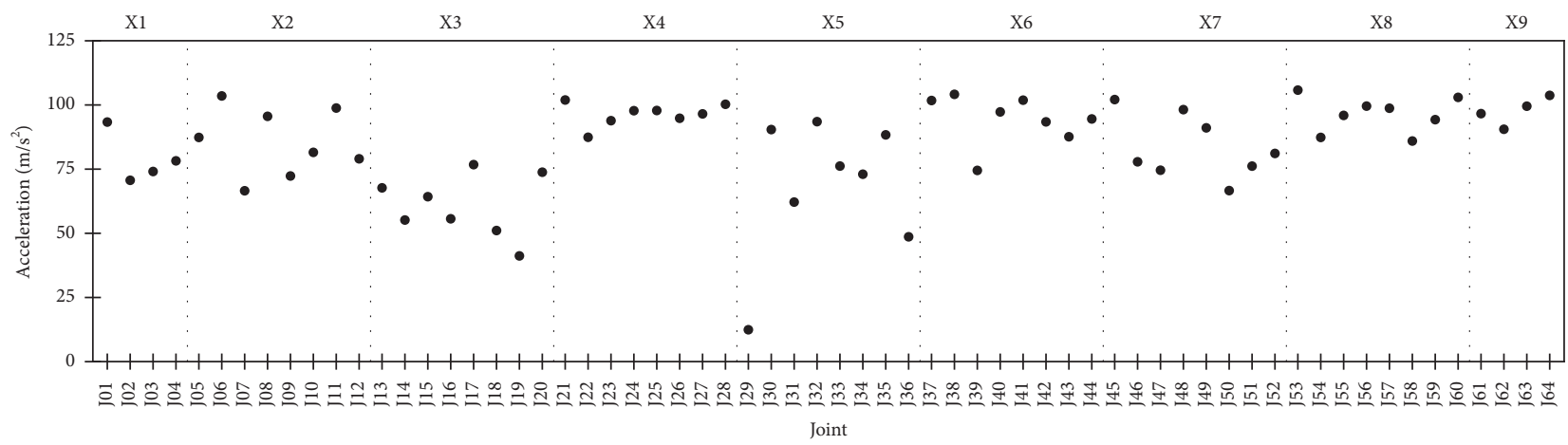

Figure 5: Average absolute peak acceleration response.

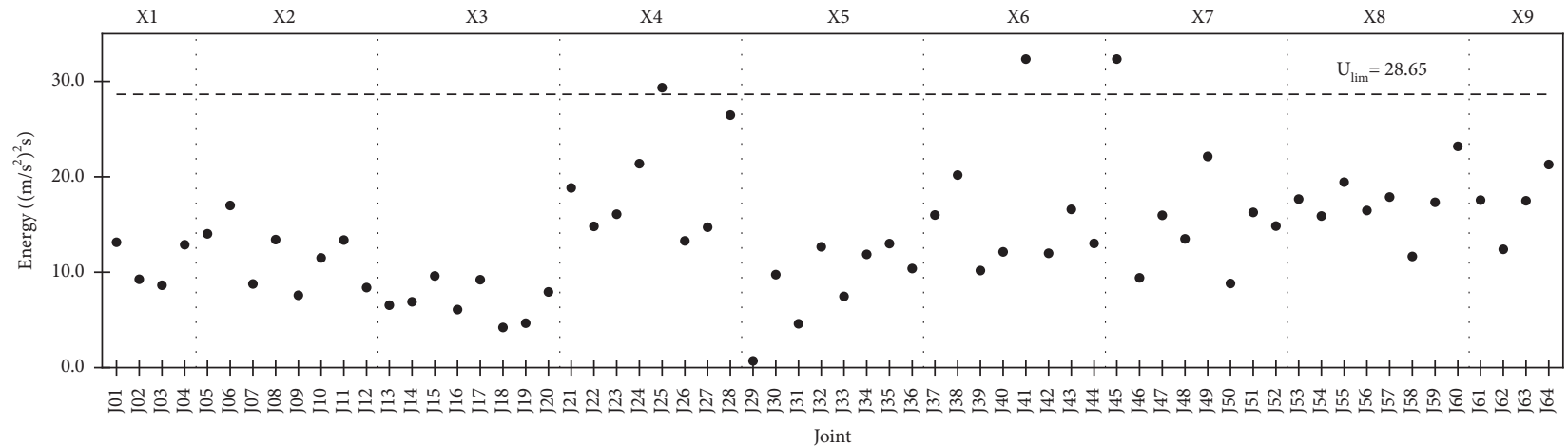

(a)

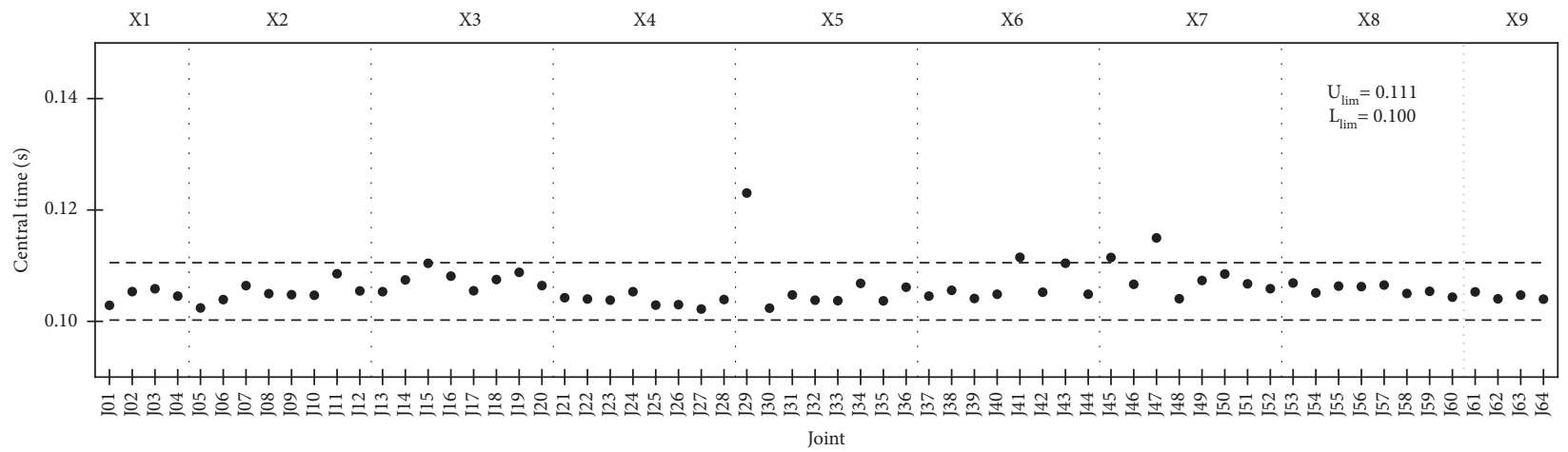

(b)

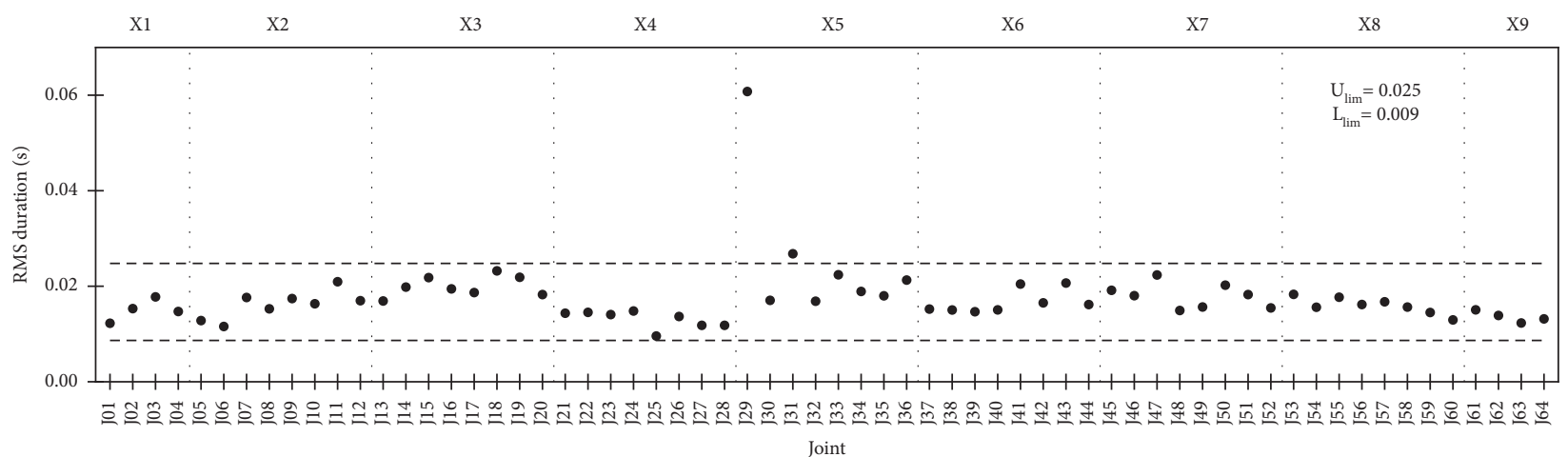

(c)

FIgURE 6: Analysis results of the central and first two normalized central moments from the average of 5 impacts. 


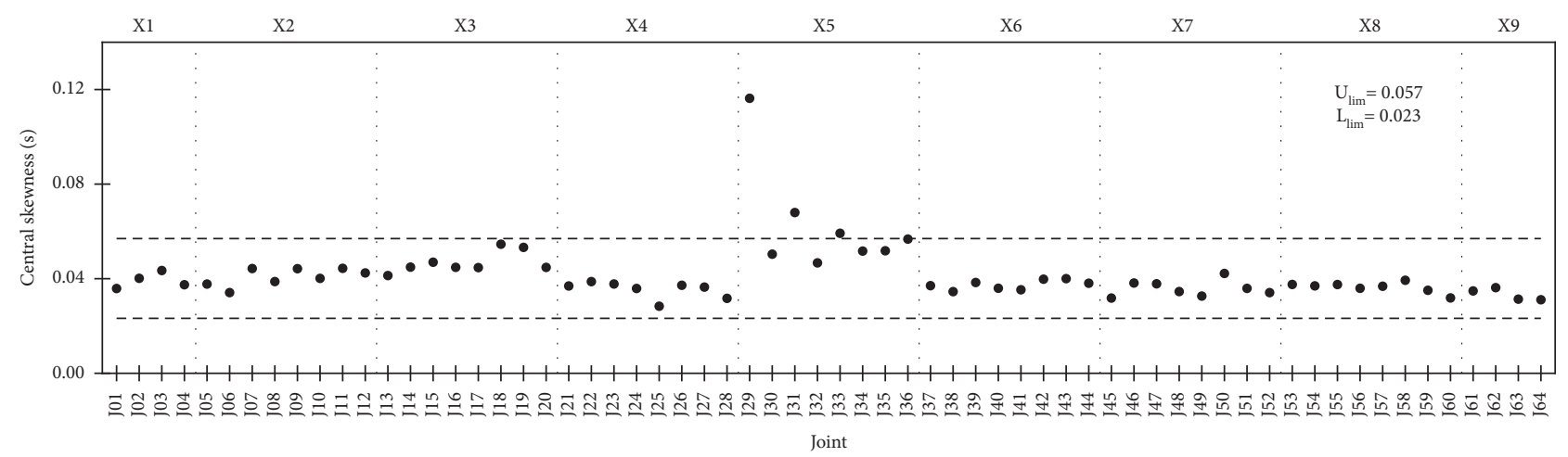

(a)

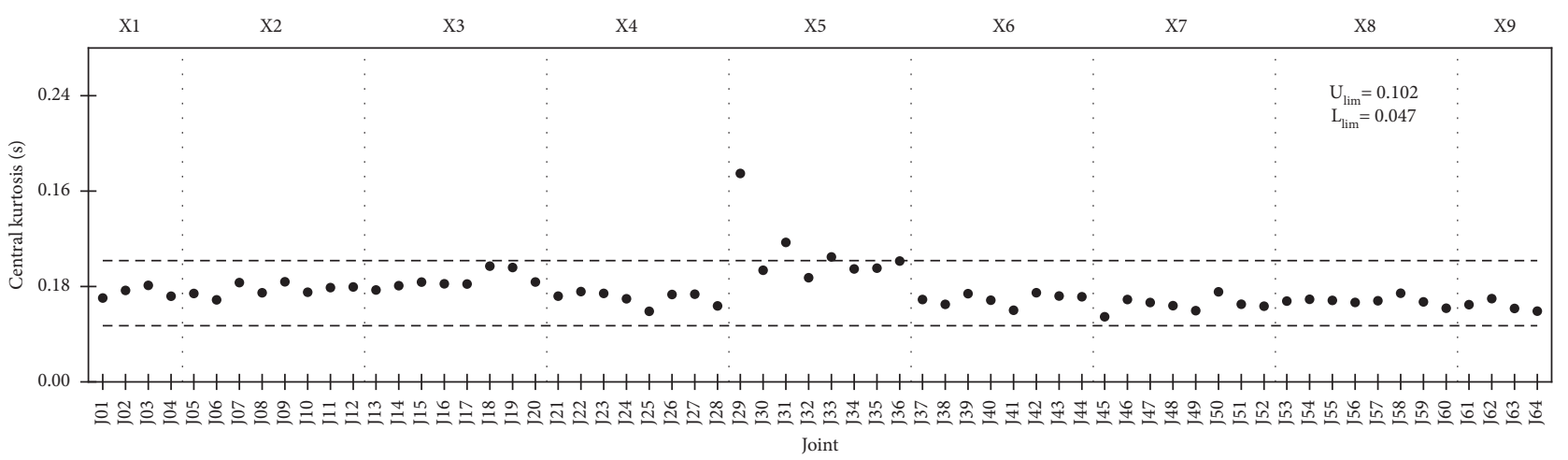

(b)

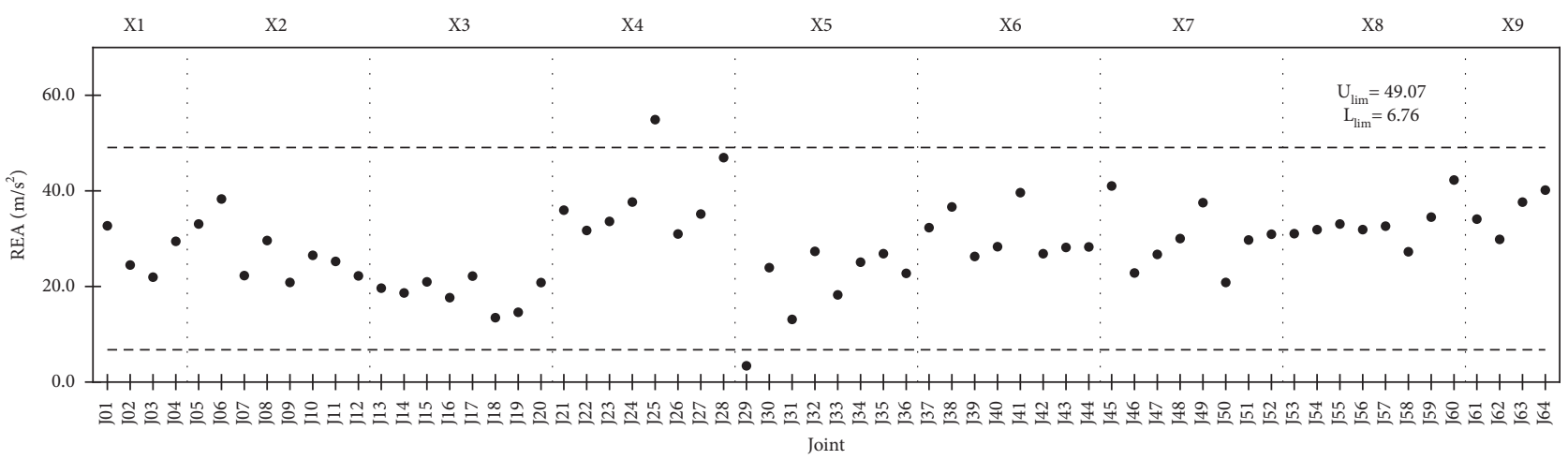

(c)

Figure 7: Analysis results of the normalized central moments and the root energy amplitude from the average of 5 impacts.

In summary, the analysis results show that the two known damaged joint connections, J29 and J31, can be identified with outliers in all and several of the statistical parameters, respectively, except for energy. Furthermore, joints J25, J33, J36, J41, and J45 have outliers in two of the statistical parameters shown. Finally, J18 and J19 have no outliers; however, these joints have values close to the outlier limits for all statistical parameters.

4.4. Damage Indicator Matrix. The damage indicator matrix provides a comparison of all joints using the statistical parameters. The damage indicator, DI, is related to the correlation coefficient, $\rho_{\mathbf{d}_{m} \mathbf{d}_{n}}$, and is defined as

$$
\mathrm{DI}=1-\rho_{\mathbf{d}_{m} \mathbf{d}_{n}}=1-\frac{\sigma_{\mathbf{d}_{m} \mathbf{d}_{n}}}{\sigma_{\mathbf{d}_{m}} \sigma_{\mathbf{d}_{n}}}
$$

Here, $\mathbf{d}_{m}$ and $\mathbf{d}_{n}$ are the feature vectors defined according to equation (16) of joints $m$ and $n$, respectively. Correspondingly, $\sigma_{\mathbf{d}_{m}}$ and $\sigma_{\mathbf{d}_{n}}$ are the standard deviations of $\mathbf{d}_{m}$ and $\mathbf{d}_{n}$, respectively, whereas $\sigma_{\mathbf{d}_{m} \mathbf{d}_{n}}$ is the covariance matrix. The damage indicator provides a nondimensional measure based on the correlation of the feature vectors. By systematically establishing damage indicators result in the damage indicator matrix; a statistical comparison of all joints based on the measure of correlation.

Correlation describes the degree of linearity, or linear dependence, between two variables or vectors. Thus, the 
TABLE 2: Detailed results of the statistical parameters for relevant joint connections.

\begin{tabular}{|c|c|c|c|c|c|c|}
\hline Joint & $E\left(\left(\mathrm{~m} / \mathrm{s}^{2}\right)^{2} \mathrm{~s}\right)$ & $T(\mathrm{~s})$ & $D(\mathrm{~s})$ & $S_{t}(\mathrm{~s})$ & $K_{t}(\mathrm{~s})$ & $A_{E}\left(\mathrm{~m} / \mathrm{s}^{2}\right)$ \\
\hline J18 & 4.21 & 0.108 & 0.023 & 0.055 & 0.097 & 13.47 \\
\hline J19 & 4.66 & 0.109 & 0.022 & 0.053 & 0.096 & 14.60 \\
\hline $\mathrm{J} 25$ & 29.35 & 0.103 & 0.010 & 0.028 & 0.059 & 54.90 \\
\hline $\mathrm{J} 29$ & 0.71 & 0.123 & 0.061 & 0.116 & 0.175 & 3.40 \\
\hline $\mathrm{J} 31$ & 4.60 & 0.105 & 0.027 & 0.068 & 0.117 & 13.11 \\
\hline J33 & 7.46 & 0.104 & 0.022 & 0.059 & 0.105 & 18.24 \\
\hline J36 & 10.39 & 0.106 & 0.021 & 0.057 & 0.101 & 22.74 \\
\hline $\mathrm{J} 41$ & 32.34 & 0.112 & 0.021 & 0.035 & 0.060 & 39.63 \\
\hline J45 & 32.34 & 0.112 & 0.019 & 0.032 & 0.055 & 41.03 \\
\hline Mean & 13.94 & 0.106 & 0.017 & 0.042 & 0.077 & 28.67 \\
\hline Median & 13.21 & 0.105 & 0.016 & 0.038 & 0.073 & 28.88 \\
\hline$L_{\lim }$ & - & 0.100 & 0.009 & 0.023 & 0.047 & 6.76 \\
\hline$U_{\lim }$ & 28.65 & 0.111 & 0.025 & 0.057 & 0.102 & 49.07 \\
\hline
\end{tabular}

damage indicator provides a measure of similarity in the behaviour of joints. If two variables are independent, the correlation coefficient is 0 , whereas it will be in the range between -1 and 1 if one of the variables is partially linearly dependent on the other. The latter depends on the strength of the linear dependence. As such, for the damage indicators, a low value will indicate similar behaviour between joints, whereas higher values will indicate a different behaviour. The majority of the joints are undamaged and exhibit similar structural behaviour; thus, these joints have similar statistical parameters. Consequently, the correlation between their respective feature vectors is expected to be close to 1 , resulting in a DI value close to 0 .

The normalized damage indicator matrix for all joints is shown in Figure 8. This figure shows that the damaged joint J29 is clearly indicated. Additionally, weak indications are observed for J31. However, indications of damage are also observed for undamaged joints, particularly J41 and J45 but also J18 and J19. The figure shows that the normalized DI values of J29 to both J41 and J45 are high, and the most obvious reason for this outcome is due to the energy. Energy $(E)$ is simply the area of the signal squared within the defined time of the acceleration response recording. Low energy implies a weak or damped transient vibration. Little or no transmissibility of the signal between the impact location and the sensor is the most obvious cause resulting in low energy. This implies that the signal must travel a longer distance before it is recorded by the sensor, and due to inherent material and structural damping, the signal amplitudes become lower resulting in a loss of energy. This phenomenon in turn provides an indication of structural damage. In contrast, high energy implies strong transient vibration.

For the joints evaluated, there is no obvious explanation for the results of high energy. Hence, a new normalized damage indicator matrix for all joints is established based on feature vectors where the energy is excluded, as shown in Figure 9. From this figure, the damaged joint J29 is clearly indicated. An indication of damage is also observed for the damaged joint J31. Furthermore, false-positive indications, i.e., indication of damage when no damage is present, are seen for joints J18 and J19. The non-normalized mean DI values of these joints are established in Table 3. The mean DI value of each joint compared to other joints identifies the joints that, on average, have feature vectors that correlate less with others.

Based on the results obtained, it is observed that (1) most of the joints are well correlated, (2) damage in a joint connection can, but must not necessarily be, well reflected in the correlation between the feature vectors, and (3) the damage indicator is not a measure of the degree of any damage. A discussion of the statistical parameters related to the structural understanding is provided in the following section to conclude on the results obtained.

\section{Discussions}

5.1. Importance of Statistical Parameters on Structural Damage Identification. The known damage in the joint connections of the bridge deck results in unwanted vertical movement and consequently a reduction in the loadbearing capacity of the stringers. Due to damage, the connectivity of the joints is changed by reduced stiffness. This, in turn, is expected to result in a change in the signal transmissibility for the joint considered between the impact location and the sensor. This change caused by damage is mainly expected to reduce the transmissibility of the signal.

As described in the previous section, low energy $(E)$ is caused by low transmissibility of the signal. The root energy amplitude $\left(A_{E}\right)$ normalizes the energy by the RMS duration and is an alternative energy feature. Low values of both energy features are expected to be caused by damaged joint connections. Central time $(T)$ measures the time at which the centroid of the energy is located. In general, the central time is expected to be located shortly after the peak of the signal occurring at $0.1 \mathrm{~s}$. There are two main explanations for obtaining high values of this feature: weak decay of a strong transient signal or generally weak transient signal behaviour. The first is not due to damage. A low local structural damping affects a strong transient signal resulting in low decay. Similarly, any reflection of signals from the surrounding structure affects 


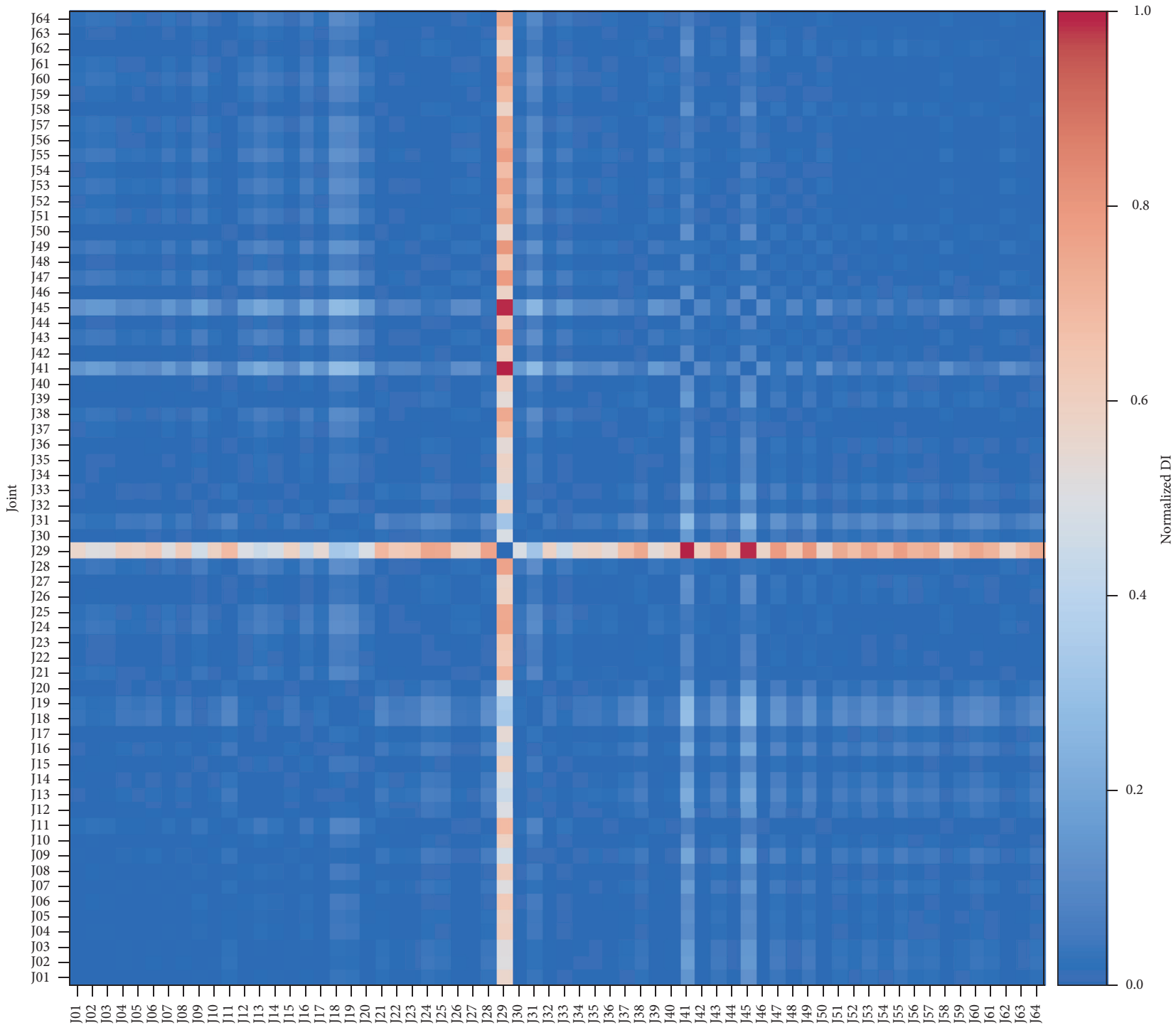

Joint

FIGURE 8: Normalized damage indicator matrix.

the transient part of the signal and consequently increases the central time. However, a generally weak transient signal behaviour is expected to be caused by damage. As such, high values for the central time can occur for both damaged and undamaged joints. RMS duration (D) measures the dispersion of the energy in a signal. Strong transient signals with high transmissibility have low dispersion, whereas weak transient signals with low transmissibility have high dispersion. Hence, RMS duration characterizes the signal well and is consequently a good feature for indicating damage. Central skewness $\left(S_{t}\right)$ describes the shape of the distribution, or the signal itself, in terms of symmetry. Regardless of the presence of damage, all joints considered will have a positive skewness because high amplitudes are located to the left of the central time with a corresponding low-amplitude tail on the right. Damaged joints, characterized by low transmissibility, will obtain a larger skewness than undamaged joints and are expected to be characterized by this feature.
The same applies for central kurtosis $\left(K_{t}\right)$, which measures the outliers in the signal energy. A high number of outliers in a signal indicates damage.

The results clearly show that RMS duration, central skewness, and central kurtosis best describe the damaged joint connections. Consequently, these are considered the most relevant features. The significance of the energy features in relation to damage is important. However, these features do not clearly describe both damages. Furthermore, the energy feature results in three outliers with high values, which is not well understood. The root energy amplitude provides better results; however, one outlier with a high value is still observed. Central time clearly indicates one damaged joint but shows outliers for undamaged joints.

By excluding energy, the remaining features can be included in the context of observing any trends that can strengthen the indication of damage. This is evident by performing an evaluation using the damage indicator matrix. 


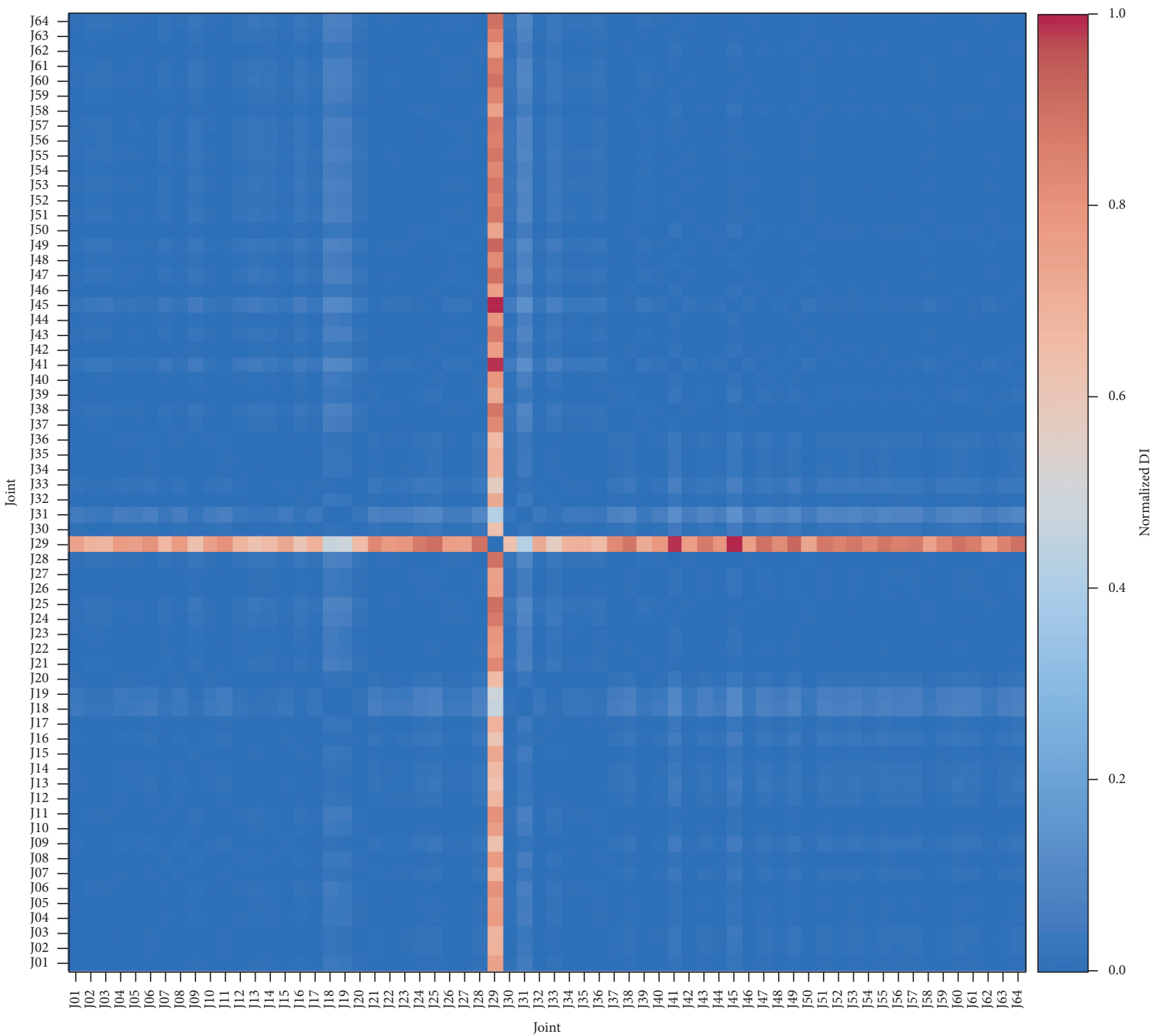

FIgURE 9: Normalized damage indicator matrix. Energy $(E)$ is excluded from the feature vector.

TABle 3: Non-normalized mean DI values.

\begin{tabular}{lc}
\hline Joint & Average DI \\
\hline J29 & 0.224 \\
J31 & 0.020 \\
J18 & 0.015 \\
J19 & 0.014 \\
\hline
\end{tabular}

5.2. Effect of Sampling Frequency. Sampling at high frequencies instead of low frequencies includes more information in the transient signals. Establishing an appropriate sampling frequency with respect to damage identification is deemed important. Hence, the effect of sampling frequency on the main statistical parameters is investigated and shown in Figure 10. In this figure, the average value of all impacts for all joint connections, including J29 and J31, are analysed and compared with the average value of J29 and J31 for different sampling frequencies. Five cases are included. The first case considers the original sampling frequency with no filter applied, whereas the other cases include the original and a systematic reduction in sampling frequency with a filter applied. Data are analysed by resampling the signals after applying an 8th order Bessel antialiasing filter at $80 \%$ of the Nyquist frequency. The ability to handle rapid changes in the signal from one value to another, which is characterized in the step response, is optimized with the Bessel filter in contrast to other antialiasing filters [32]. This characteristic is favoured for the analysis of transient vibration in the time domain. However, the Bessel filter reduces the amplitudes in the passband due to the roll-off quality of the filter, which explains the reduction in results for energy and root energy amplitude from a sampling frequency of $2048 \mathrm{~Hz}$ with no filter to $2048 \mathrm{~Hz}$ with a filter applied.

The absolute value of the percentage change in the damaged joints to the value of all joints is summarized in Table 4. Figure 10 and this table show that lowering the 


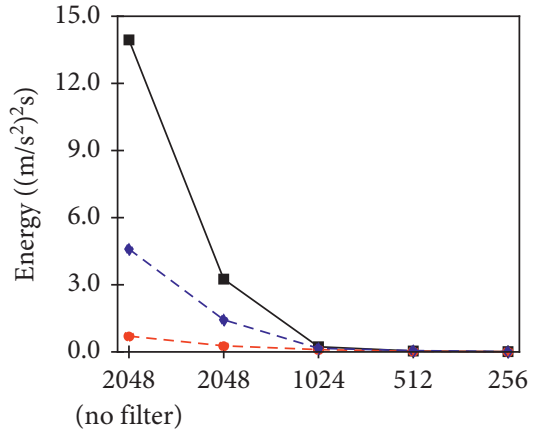

Sampling frequency $(\mathrm{Hz})$

$\rightarrow$ All joints

$-\bullet$ J29

$-\downarrow \mathrm{J} 31$

(a)

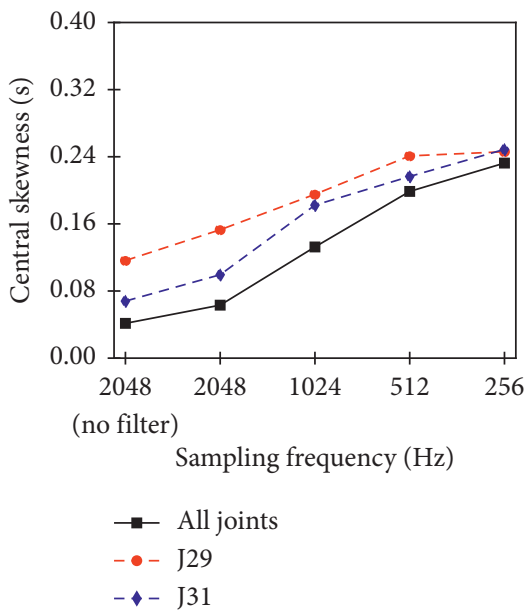

(d)

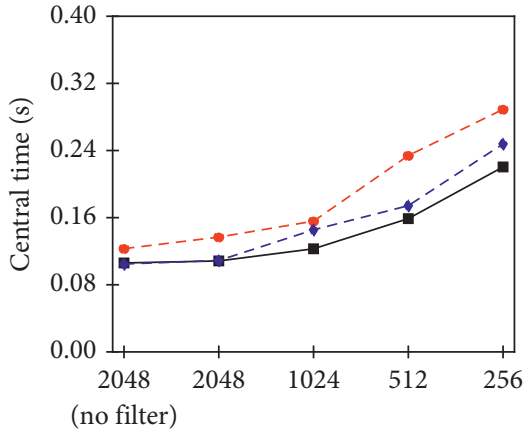

Sampling frequency $(\mathrm{Hz})$

$\rightarrow$ All joints

- $-\mathrm{J} 29$

$-\downarrow$ J31

(b)

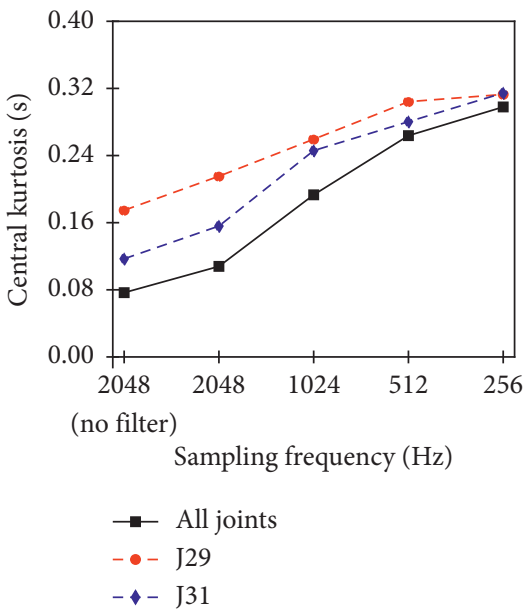

(e)

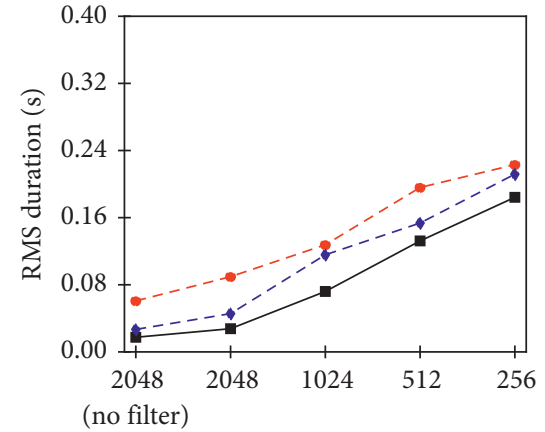

Sampling frequency $(\mathrm{Hz})$

$\rightarrow$ All joints

$-\bullet$ J29

- J 31

(c)

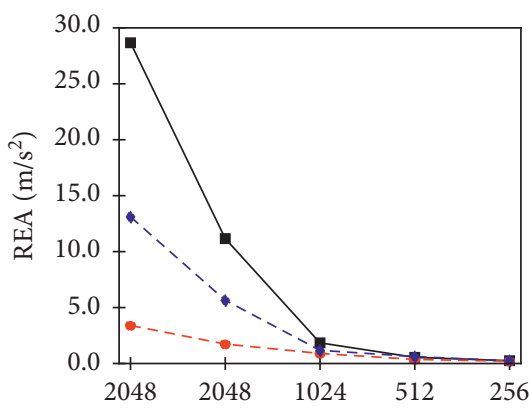

(no filter)

Sampling frequency $(\mathrm{Hz})$

$\rightarrow-$ All joints

- - J29

$-\uparrow \mathrm{J} 31$

(f)

FIgURE 10: Analysis results for different sampling frequencies.

TABLE 4: Absolute value of percentage change in the damaged joints to the value of all joints.

\begin{tabular}{|c|c|c|c|c|c|c|}
\hline \multirow{2}{*}{ Statistical parameter } & \multirow{2}{*}{ Joint } & \multicolumn{5}{|c|}{ Percentage change for different sampling frequencies (\%) } \\
\hline & & $2048 \mathrm{~Hz}$ (no filter) & $2048 \mathrm{~Hz}$ & $1024 \mathrm{~Hz}$ & $512 \mathrm{~Hz}$ & $256 \mathrm{~Hz}$ \\
\hline \multirow{2}{*}{ E } & $J 29$ & 95 & 92 & 55 & 35 & 25 \\
\hline & $\mathrm{J} 31$ & 67 & 56 & 30 & 48 & 0 \\
\hline \multirow{2}{*}{$T$} & $\mathrm{~J} 29$ & 16 & 27 & 27 & 47 & 31 \\
\hline & $\mathrm{J} 31$ & 1 & 1 & 19 & 9 & 13 \\
\hline \multirow{2}{*}{$D$} & $\mathrm{~J} 29$ & 259 & 221 & 78 & 48 & 21 \\
\hline & $\mathrm{J} 31$ & 59 & 64 & 61 & 17 & 15 \\
\hline \multirow{2}{*}{$S_{t}$} & $\mathrm{~J} 29$ & 176 & 143 & 47 & 21 & 6 \\
\hline & $\mathrm{J} 31$ & 62 & 57 & 37 & 9 & 7 \\
\hline \multirow{2}{*}{$K_{t}$} & $\mathrm{~J} 29$ & 127 & 99 & 34 & 15 & 5 \\
\hline & $\mathrm{J} 31$ & 52 & 44 & 27 & 6 & 6 \\
\hline \multirow{2}{*}{$A_{E}$} & $\mathrm{~J} 29$ & 88 & 84 & 51 & 34 & 18 \\
\hline & $\mathrm{J} 31$ & 54 & 49 & 36 & 13 & 4 \\
\hline
\end{tabular}

sampling frequency in general decreases the identifiability of known damaged joints from the average of all joints when considering the statistical parameters, except for central time. Information is lost for the resampled signals. This lost information in turn provides a weaker indication of damage.
Lower sampling frequencies result in fewer high amplitudes in the signal representations. This is clearly seen when considering the analysis results of the energy features. The central time increases, and the centroid of the signals moves away from $0.1 \mathrm{~s}$. In general, the dispersion of the signals 
increases with lower sampling frequencies, and the distinction of damaged joint connections appears to be unaffected. However, this is not the case when considering the absolute value of the percentage change for the RMS duration, which shows that the percentage change is lowered with decreasing sampling frequency. For central skewness and central kurtosis, it is apparent that the identification of damage is more difficult with lower sampling frequencies both when considering the analysis results and the absolute value of percentage change.

By considering the parameters most relevant for the identification of damage, it is clearly seen that a reduction in sampling frequency reduces the identifiability of damage, except when considering central time. A higher sampling frequency provides a better representation of the transient signals and includes more information. The values of the statistical parameters strongly depend on the sampling frequency and, as such, can only be used for the purpose of comparison.

The obtained results show that an adequate sampling frequency is needed to identify damage. A minimum sampling frequency of $2048 \mathrm{~Hz}$ is recommended for this case. However, it should be noted that using lower sampling frequencies does not exclude the possibility of identifying damage.

5.3. Summary. Experience obtained from the experimental study leads to the following general observations:

(i) There are two known damaged joint connections, J29 and J31. From the proposed methodology, a clear identification of J29 is established, whereas a weak indication of $\mathrm{J} 31$ is observed. Although the methodology identifies both damaged joint connections, the distinction between J31 and the undamaged joint connections J18 and J19 is low, resulting in two false-positive indications of damage.

(ii) When considering the statistical parameters only, the RMS duration, central skewness, and central kurtosis are of primary interest. Additionally, root energy amplitude is important due to its significance related to the damage configuration studied.

(iii) The damage indicator matrix provides a statistical comparison of all joints based on the measure of correlation using feature vectors. From the results obtained, three undamaged joint connections obtained high energy. No reasonable explanation of this phenomenon is found. When excluding this statistical parameter from the feature vectors, an improved damage indicator matrix is obtained.

(iv) A high sampling frequency is required. High sampling frequency provides better results and more clear identification of damage due to increased information included in the signals analysed.

(v) An adequate number of joint connections is needed to obtain a basis for comparison. A high number of identical joints forming the baseline provides a more reliable result. This is clearly observed when comparing results from one impact location to the results of all impact locations.

(vi) The possibility that changes in the statistical parameters are caused by inconsistent input, i.e., excitation, cannot be eliminated. Inconsistent input can be limited to the best extent possible by using the average of multiple impacts. The results obtained in this study show that using 5 impacts per impact location is considered adequate for this purpose.

The proposed methodology establishes damaged joint connections in the bridge deck and is based on the transmissibility of the signal from the impact location to the sensor and through the joint connection to be assessed. All joint connections have the same loadbearing function. The location of any damaged joints, or the distance between any damaged joints, does not affect the indication of damage since the evaluation is based on local response and not on the global response of the structure. Damaged joint connections are identified by comparing statistical parameters based on temporal moments to nominally identical connections. The damaged joint connections are localized utilizing the instrumentation setup and a systematic monitoring procedure. The baseline is established from the statistical parameters evaluated for all joint connections. The observation made in the first bullet point (i) leads to the conclusion that the methodology can only clearly identify one damaged joint connection. However, from an inspector's perspective, such a result should lead to the further investigation of all four joints with the assumption of damage being present. Further investigation should be to carry out a full visual inspection or consider a long-term SHM system using strain measurements for bridges in operation.

\section{Conclusion}

This paper presented a new methodology for detecting damage in stringer-to-girder connections from the bridge deck of existing steel bridges. The methodology is based on combining the use of temporal moments to establish statistical parameters from response measurements with an appropriate instrumentation setup and a systematic monitoring procedure. An experimental study on a full-scale steel bridge identified and localized two damaged joint connections in the bridge deck by (1) investigating statistical parameters from response measurements and (2) establishing a damage indicator matrix by comparing feature vectors using correlation analysis. The importance of high sampling frequency to obtain the best possible identification of damaged joint connections was shown through a sensitivity analysis. A minimum sampling frequency of $2048 \mathrm{~Hz}$ is recommended for similar applications.

The main limitation of the methodology presented herein is the need for an adequate number of joint connections or similar structural components to obtain a baseline for comparison. The baseline for comparison can be 
affected in cases where many damages are present in the structural system. Nevertheless, the methodology presented in this paper demonstrates that structural damage in joint connections can be effectively established. The proposed methodology is easy to implement, both in a technical and practical manner. Limited technical equipment is needed. Furthermore, this methodology can be applied to components that are difficult to access without the need for temporary installation of access support. As such, the established methodology can contribute to improving the identification of critical damage during scheduled inspection of existing open-deck highway and railway bridges in service with no, or limited, downtime. Further investigation should be to (1) test the methodology on bridges in service and (2) use the established methodology but consider structural response obtained from vehicles on damage detection for potential automation purposes.

\section{Data Availability}

The data used to support the findings of this study are available from the corresponding author upon request.

\section{Conflicts of Interest}

The authors declare that there are no conflicts of interest regarding the publication of this paper.

\section{Acknowledgments}

The Hell Bridge Test Arena is financially supported by the Norwegian Railway Directorate and Bane NOR.

\section{References}

[1] R. Haghani, M. Al-Emrani, and M. Heshmati, "Fatigue-prone details in steel bridges," Buildings, vol. 2, no. 4, pp. 456-476, 2012.

[2] B. Åkesson, Fatigue Life of Riveted Steel Bridges, Chalmers University of Technology, Gothenburg, Sweden, 1994.

[3] D. H. Tobias and D. A. Foutch, "Reliability-based method for fatigue evaluation of railway bridges," Journal of Bridge Engineering, vol. 2, no. 2, pp. 53-60, 1997.

[4] J. D. DiBattista, D. Adamson, and G. L. Kulak, "Evaluation of remaining fatigue life for riveted truss bridges," Canadian Journal of Civil Engineering, vol. 25, no. 4, pp. 678-691, 1998.

[5] T. E. Cousins, J. M. Stallings, D. A. Lower, and T. E. Stafford, "Field evaluation of fatigue cracking in diaphragm-girder connections," Journal of Performance of Constructed Facilities, vol. 12, no. 1, pp. 25-32, 1998.

[6] C. W. Roeder, G. MacRae, P. Crocker, K. Arima, and S. Wong, "Dynamic response and fatigue of steel tied-arch bridge," Journal of Bridge Engineering, vol. 5, no. 1, pp. 14-21, 2000.

[7] B. M. Imam, T. D. Righiniotis, and M. K. Chryssanthopoulos, "Probabilistic fatigue evaluation of riveted railway bridges," Journal of Bridge Engineering, vol. 13, no. 3, pp. 237-244, 2008.

[8] T. Guo and Y.-W. Chen, "Field stress/displacement monitoring and fatigue reliability assessment of retrofitted steel bridge details," Engineering Failure Analysis, vol. 18, no. 1, pp. 354-363, 2011.

[9] T. Guo, D. M. Frangopol, and Y. Chen, "Fatigue reliability assessment of steel bridge details integrating weigh-in-motion data and probabilistic finite element analysis," Computers \& Structures, vol. 112-113, pp. 245-257, 2012.

[10] M. Al-Emrani, "Fatigue performance of stringer-to-floorbeam connections in riveted railway bridges," Journal of Bridge Engineering, vol. 10, no. 2, pp. 179-185, 2005.

[11] M. Al-Emrani, B. åkesson, and R. Kliger, "Overlooked secondary effects in open-deck truss bridges," Structural Engineering International, vol. 14, no. 4, pp. 307-312, 2004.

[12] M. Al-Emrani and R. Kliger, "FE analysis of stringer-to-floorbeam connections in riveted railway bridges," Journal of Constructional Steel Research, vol. 59, no. 7, pp. 803-818, 2003.

[13] B. M. Imam, M. K. Chryssanthopoulos, and D. M. Frangopol, "Fatigue system reliability analysis of riveted railway bridge connections," Structure and Infrastructure Engineering, vol. 8, no. 10, pp. 967-984, 2012.

[14] T. D. Righiniotis, B. M. Imam, and M. K. Chryssanthopoulos, "Fatigue analysis of riveted railway bridge connections using the theory of critical distances," Engineering Structures, vol. 30, no. 10, pp. 2707-2715, 2008.

[15] B. M. Imam, T. D. Righiniotis, and M. K. Chryssanthopoulos, "Numerical modelling of riveted railway bridge connections for fatigue evaluation," Engineering Structures, vol. 29, no. 11, pp. 3071-3081, 2007.

[16] B. Imam, T. D. Righiniotis, M. K. Chryssanthopoulos, and B. Bell, "Analytical fatigue assessment of riveted rail bridges," Proceedings of the Institution of Civil Engineers - Bridge Engineering, vol. 159, no. 3, pp. 105-116, 2006.

[17] I. Olofsson, L. Elfgren, B. Bell et al., "Assessment of European railway bridges for future traffic demands and longer lives-EC project "sustainable bridges"," Structure and Infrastructure Engineering, vol. 1, no. 2, pp. 93-100, 2005.

[18] C. R. Farrar and K. Worden, "An introduction to structural health monitoring," Philosophical Transactions of the Royal Society A, vol. 365, no. 1851, pp. 303-315, 2007.

[19] S. W. Doebling, C. R. Farrar, M. B. Prime, and D. W. Shevitz, "Damage identification and health monitoring of structural and mechanical systems from changes in their vibration characteristics: a literature review," Report LA-13070-MS, Los Alamos National Laboratory, New Mexico, NM, USA, 1996.

[20] H. Sohn, C. R. Farrar, F. M. Hemez et al., "A review of structural health monitoring literature: 1996-2001,” Report LA-13976-MS, Los Alamos National Laboratory, New Mexico, NM, USA, 2004.

[21] H. R. Ahmadi, F. Daneshjoo, and N. Khaji, "New damage indices and algorithm based on square time-frequency distribution for damage detection in concrete piers of railroad bridges," Structural Control and Health Monitoring, vol. 22, no. 1, pp. 91-106, 2015.

[22] H. R. Ahmadi and D. Anvari, "New damage index based on least squares distance for damage diagnosis in steel girder of bridge's deck," Structural Control and Health Monitoring, vol. 25, no. 10, pp. 1-22, 2018.

[23] M. Bayat, H. R. Ahmadi, and N. Mahdavi, "Application of power spectral density function for damage diagnosis of bridge piers," Structural Engineering and Mechanics, vol. 71, no. 1, pp. 57-63, 2019.

[24] D. O. Smallwood, "Characterization and simulation of transient vibrations using band limited temporal moments," Shock and Vibration, vol. 1, Article ID 940635, 21 pages, 1994.

[25] F. M. Hemez and S. W. Doebling, "From shock response spectrum to temporal moments and vice-versa," Report LAUR-02-6790, Los Alamos National Laboratory, New Mexico, NM, USA, 2002.

[26] C. R. Farrar and K. Worden, Structural Health Monitoring: A Machine Learning Perspective, John Wiley \& Sons, Hoboken, NJ, USA, 2012. 
[27] B. T. Svendsen, "bjorntsv/tempmom," 2020.

[28] K. Worden and J. M. Dulieu-Barton, "An overview of intelligent fault detection in systems and structures," Structural Health Monitoring: An International Journal, vol. 3, no. 1, pp. 85-98, 2004.

[29] A. Rytter, Vibrational Based Inspection of Civil Engineering Structures, University of Aalborg, Aalborg, Denmark, 1993.

[30] K. Worden, C. R. Farrar, G. Manson, and G. Park, "The fundamental axioms of structural health moitnoring," Proceedings of the Royal Society A: Mathematical, Physical and Engineering Sciences, vol. 463, no. 2082, pp. 1639-1664, 2007.

[31] E. Kreyszig, Advanced Engineering Mathematics, John Wiley \& Sons, Hoboken, NJ, USA, 9th edition, 2006.

[32] S. Smith, Digital Signal Processing: A Practical Guide for Engineers and Scientists, Newness, Oxford, UK, 2002. 